\title{
Low-cost Automation for Prepreg Handling - Two Cases from the Aerospace Industry
}

\author{
Andreas Björnsson, Jan Erik Lindbäck, Daniel Eklund and Marie Jonsson
}

\section{Linköping University Post Print}

\section{Tweet}

N.B.: When citing this work, cite the original article.

Original Publication:

Andreas Björnsson, Jan Erik Lindbäck, Daniel Eklund and Marie Jonsson, Low-cost Automation for Prepreg Handling - Two Cases from the Aerospace Industry, 2015, SAE International Journal of Materials and Manufacturing, (9), 1.

http://dx.doi.org/10.4271/2015-01-2606

Copyright: 2015 SAE International.

http://papers.sae.org/

Postprint available at: Linköping University Electronic Press

http://urn.kb.se/resolve?urn=urn:nbn:se:liu:diva-121602 


\title{
Low-cost Automation for Prepreg Handling - Two Cases from the Aerospace Industry
}

\author{
Author, co-author (Do NOT enter this information. It will be pulled from participant tab in \\ MyTechZone) \\ Affiliation (Do NOT enter this information. It will be pulled from participant tab in MyTechZone)
}

\begin{abstract}
With an increased use of composite materials within the aerospace industry follows a need for rational and cost-effective methods for composite manufacturing. Manual operations are still common for low to medium manufacturing volumes and complex products. Manual operations can for example be found in material handling, when picking prepreg plies from a cutter table and stacking them to form a plane laminate in preparation for a subsequent forming operation. Stacking operations of this kind often involves a great number of different ply geometries and removal of backing paper and other protecting materials like plastic. In this paper two different demonstrator cells for automated picking of prepreg plies and stacking of plane laminates are presented. One demonstrator is utilizing a standard industrial robot and an advanced end-effector to handle the ply variants. The other demonstrator is using a dual arm robot which allow for simpler end-effector design. In combination with a previously developed system for automated removal of backing papers both systems have shown to be capable of automatically picking prepreg plies from a plane surface and stack them to generate a flat multistack laminate. The dual arm approach has shown advantageous since it result in simpler end-effector design and a successive lay down sequence that result in good adhesion between the plies in the laminate.
\end{abstract}

\section{Introduction}

The combination of high strength and low weight that is achievable with composite materials like Carbon Fiber Reinforced Plastic (CFRP) has made these types of materials attractive to the aerospace sector. The use of CFRP in aircrafts has steadily increased and modern aircrafts can consist of around $50 \%$ (of structural weight) of CFRP and other composite materials [1]. This entails an increase in manufacturing volumes, making rational, cost-efficient manufacturing processes an important area for further research and development.

Unidirectional (UD) carbon fiber prepreg is a commonly used high performance material in aerospace applications. However, much of the current efforts in automated handling within composite manufacturing seem to be directed towards the handling of dry fiber fabrics for preform manufacturing. There are several examples of both research groups and companies developing solutions for dry fabric handling. Within the research community there are systems that have been developed for only handling two dimensional objects
[2] as well as systems for three dimensional handling that also include draping capabilities [3] [4]. As a commercial example Broetje Automation has developed a commercial system that uses vacuum technology for lifting permeable textiles [5].

When it comes to automated handling of prepreg Ward et al. [6] conclude that concepts for automated prepreg handling have been attempted but have so far made little impact in the composite manufacturing industry. Concepts have been developed in research projects, for example in the ARMATURE project [7], and are also promoted by commercial cutting equipment providers as reported by Black [8]. However, the implementation of these concepts in full scale manufacturing operations seems to be lacking. A key issue to address in order to design automated solutions for handling prepreg materials is to design the systems so that they can handle a great variety of geometrical shapes at the same time as the system remain as uncomplicated as possible [9].

Manufacturing using UD prepreg is mainly done by one of two approaches. The first approach is to place the prepreg onto a mold with the final product shape, thus forming/draping the prepreg layer by layer. Another is to stack several prepreg layers to form a multilayer laminate (multistack) before giving the product its final shape it in a separate forming process. The handling operation of pick-up, transport and laydown of cut prepreg plies from one surface to another is in this paper defined as pick-and-place. The technical development in this paper focuses in the pick-and-place process, with the aim of providing low-cost solution suitable for low-to-medium manufacturing volumes.

For composite manufacturing where prepreg is laid up directly on a mold tool manual operations are common for low production volumes and for complex parts. In manual layup the two major tasks, cutting and layup of prepreg plies, can account for around $40-60 \%$ of the manufacturing costs of a part [10]. For more complex shapes such as double curved surfaces or sharp corners it is difficult to lay down a ply correctly on the mold the first time without having to make small adjustments [12]. By using a manufacturing approach with a layup of a flat laminate, a multistack, followed by a forming operation the layup operation be simplified since it reduces the layup from a three dimensional case to a two dimensional case. A simplified layup case is especially advantageous when considering automated solutions since the end-effector does not have to conform to a curvature when placing a stack or a ply.

Page 1 of 7 
Automated Tape Laying (ATL) and Automated Fiber Placement (AFP) are absolutely dominant when it comes to automated layup of prepreg onto a mold [11]. However these two technologies have limitations in what parts can be manufactured cost efficiently. Geometrically complex parts with, for example short lay-down lengths and sharp corners and radii, can be difficult to manufacture using AFP or ATL resulting in a slow manufacturing process. There are cases, also when considering layup of flat multistacks, where ATL and AFP cannot be used due to required geometries and stacking sequences.

All in all, there is a need for automation targeting low to medium manufacturing volumes of complex prepreg based structures, where manual pick-an-place operations today induces a large part of the cost.

\section{Prepreg handling and gripper technologies}

According to Buckingham and Newell [12] the two key material properties that affect the handling of prepreg are the rigidity and the tack. In composite manufacturing tack is usually defined as the ability of two prepreg plies to adhere to each other [13]. Unidirectional materials are sensitive to damages due to their highly anisotropic nature. The backing papers that are commonly used to protect prepreg also help to improve the rigidity of prepreg materials [12]. However, keeping the backing paper during handling operations to provide protection and improved rigidity requires a process to peel off the backing paper after handing. The peeling has been identified as a difficult process [12] but automated solutions have been developed and tested with promising results [14].

The tack of the material is related to the resin type, the fiber-to-resin ratio, the distribution of the resin and the temperature [12]. In probe tack tests Dubois et al. [15] show that the adhesion (debonding force) between unidirectional prepreg and an aluminum probe is dependent on the contact time and force between the two surfaces. Longer contact times and higher contact forces result in a stronger bond. The probe tack test also shows that the resin viscosity is reduced with increased probe temperature, resulting in an increased prepreg tack with increased temperature. However the tack response to changes in temperature is dependent on the type of material. Findings reported by Crossley et al. [16] show that some prepreg materials experience increased tack with increased temperature while others show reduced tack when the temperature is increased.

The bonding behavior of a prepreg material, affected by for example contact time and pressure as well as tackiness, can affect the release of prepreg from a gripper or from the pickup surface as well as the adhesion between prepreg and backing paper. In handling unprotected prepreg with direct contact between the prepreg and the gripper, resin can be transferred risking contamination and resin buildup and subsequent gripper failure. All of these aspects (backing paper removal, tackiness, contamination risks, different handling properties in different fiber directions etc.) make prepreg challenging for handling operations using a pick-and-place approach.

Over the years a great number of gripping technologies have been developed, some for general purposes and other for very specific tasks. In reviewing gripping technologies it can be concluded that all technologies have their merits and suitable usages as well as drawbacks and it is important to find a gripping technology that best suits the particular handling task. Gripping technologies relevant for prepreg handling are shown in Figure 1 [14].

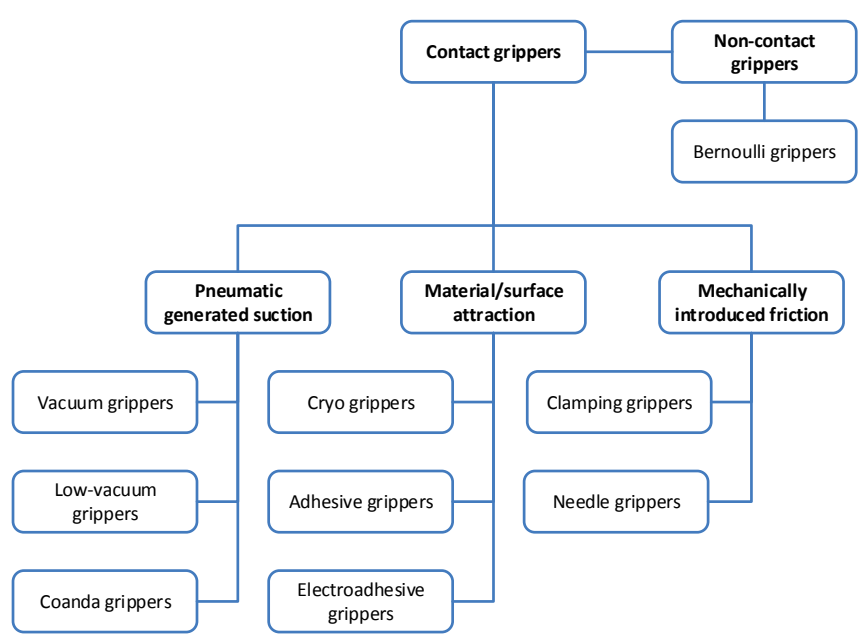

Figure 1: An overview of gripping technologies from [14].

Several of the gripping technologies introduce risks affecting the final quality, like cryo grippers that can introduce fluids [17], adhesive grippers that rely on attaching and detaching through adhesives and needle grippers that risk separating fibers. Some, like electrostatic grippers can drive system complexity [18] and others, like clamping grippers, generally require access from two sides. Coanda grippers, where the material floats under the gripper, seem to be an appealing alternative but the technology has shown problems in positional accuracy in handling operations [19]. The most common grippers in handling operations are different types of vacuum based grippers where high vacuum grippers are used to handle permeable materials, for example dry fiber fabrics, an low vacuum grippers to handle more airtight materials like prepreg.

\section{Ply stacking and stagger}

One of the more difficult aspects of automated systems for pick-andplace of prepreg is to handle the amount of different shapes that often is needed to stack a laminate, even in a flat two-dimensional case. In order to achieve desired strength and stiffness and to cancel any distortion, curvatures and avoid residual stresses of the cured product, each layer in a laminate is placed with a specific fiber angle. Also the prepreg material (or roll) often has a fixed width before cut. This means that in order to achieve the desired fiber angles for each layer, the prepreg sheet has to be cut into plies fitting the roll width, and the resulting plies assembled so that fibers run continuously in the desired angle, covering the desired stack form, see Figure 2.
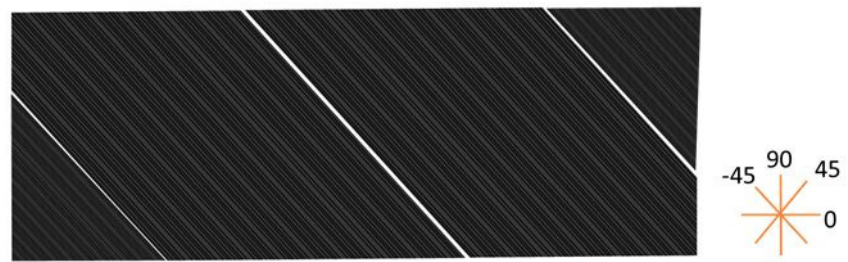

Figure 2: Example of an assembled layer out of $\mathbf{- 4 5}$ degree plies.

In order to maintain strength and stiffness properties throughout the laminate these "cut lines" cannot be repeated in the same place, thus a "stagger" is needed that shifts the cut lines, see Figure 3. Staggers

Page 2 of 7 
increase the amount of geometrical shapes in a stack, making it a challenge for cost-effective and robust gripper design.

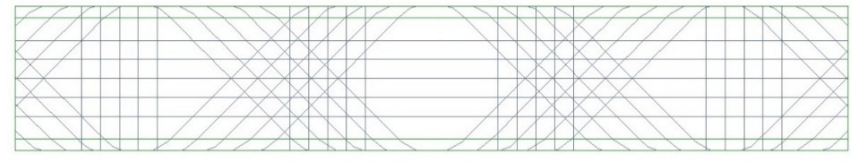

Figure 3: An example of a 40 layer stacked laminate with ply stagger. All lines of the full stack are indicated.

\section{Two Pick-and-place Demonstrators}

Automated handling of prepreg has been the focus of two separate research projects. Within each project physical demonstrators, have been built using two different approaches to the pick-and-place endeffector design. The purpose have been, experimentation and demonstration of different technical solutions. The two demonstrators are presented below and then compared as to draw conclusions in the following section.

\section{Single Manipulator Demonstrator}

This demonstrator is a demonstrator cell for automated cutting, stacking and forming of multistacked prepreg. It was developed in the Triple Use project by Swerea SICOMP, SAAB Aerostructures and KTH. The cell, shown in Figure 4 consists of a handling robot, an ABB 6700-205 with a lifting capacity of $200 \mathrm{~kg}$ and a reach of 2.85 $\mathrm{m}$. The handling robot cuts prepreg on a cutting table, picks and places the prepreg on the stack and removes the protective backing paper. The smaller robot, a KUKA KR10 R1100, with a handling capacity of $10 \mathrm{~kg}$ and a reach of $1.1 \mathrm{~m}$ then forms the multistack over a tool to produce a beam.
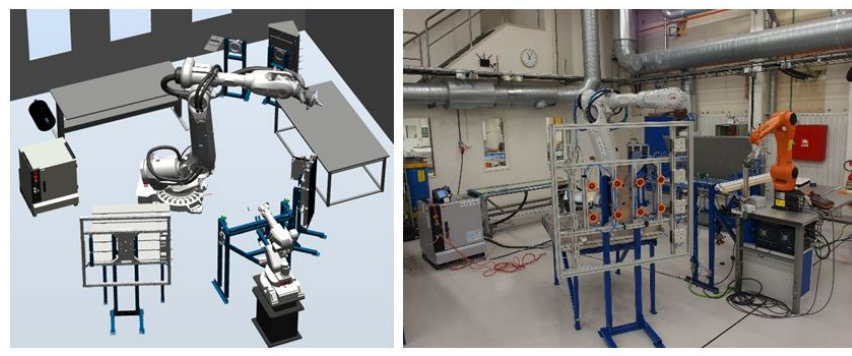

Figure 4: The demonstrator cell with a single manipulator solution. Left: the virtual cell in RobotStudio with the handling robot at the center of the cell. Right: The physical demonstrator cell.

The forming process, which has been developed by KTH as part of the project, is further described in [20]. After forming, the larger handling robot assembles the demonstrator product consisting of the formed beams and a skin. After this the product is bagged and cured making it an integrated composite structure. All manufacturing steps are described in Figure 5 but for this paper, the focus will be on the pick-and-place aspects the demonstrator cell.

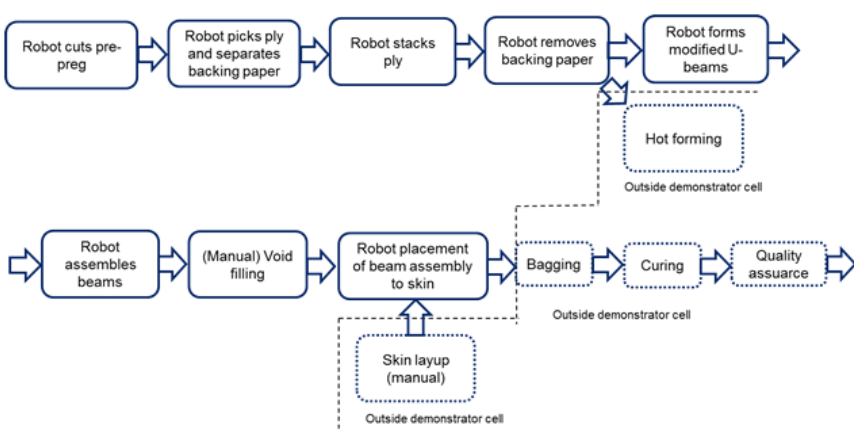

Figure 5: The manufacturing process in the demonstrator cell shown in Figure 4.

The stack handled in the described cell is shown in Figure 3 and the final gripper is shown in Figure 6. The largest plies where approximately $1100 \times 200 \mathrm{~mm}$ and the smallest around $80 \times 80 \mathrm{~mm}$. The gripper consisted of a $\mathrm{V}$-shaped frame at 45-degree angle and individual zones that can be activated depending on ply geometry. The zones are by default extended but when triggered they are retracted using a pneumatic cylinder, as illustrated in Figure 7 . This feature is used to assemble gripping zones fitting a certain ply geometry to avoid disturbing adjacent plies during picking and placing. It also allows for the backing paper separation process to get access to the ply.
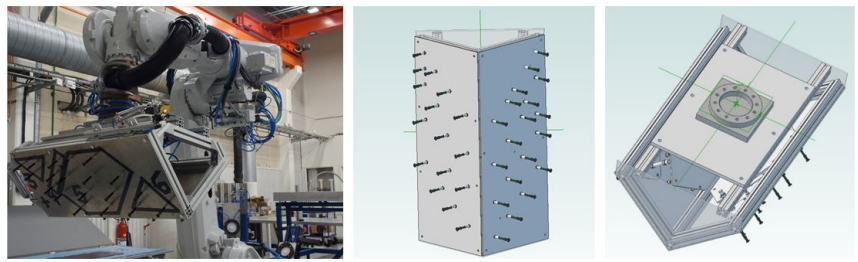

Figure 6: Left: The end-effector mounted on the robot. Zones 3 to 6 are outlined on the gripper. Middle and right: CAD-geometry, showing the two sides and the vacuum cup interfaces.

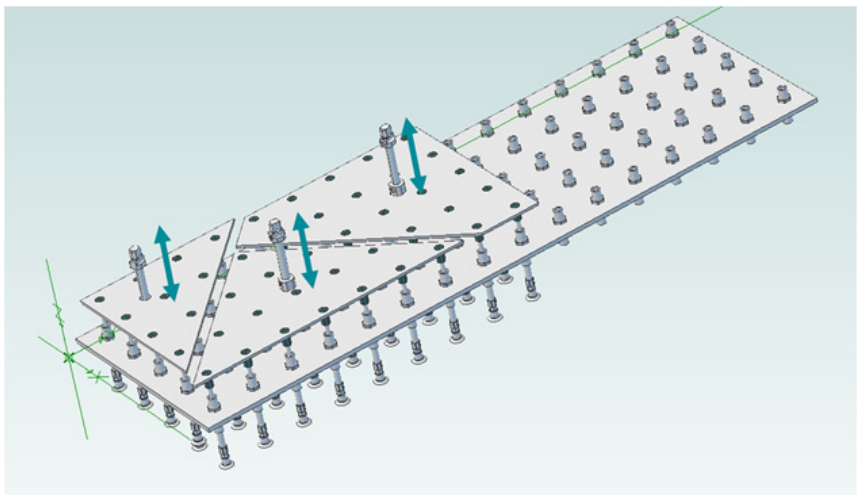

Figure 7: Example of gripper zones. Zones are extracted or retracted using pneumatic cylinders and thus "assembled" to fit different ply geometries.

At first the full stagger was considered resulting in the gripper outline shown in Figure 8 consisting of 18 zones handling 22 different ply geometries ( 0 degree layers excluded). As this resulted in a large, very complex gripper it was decided to reduce stack complexity. This 
is not possible in a commercial product, as it affects the strength and stiffness, but it was sufficient for the demonstrator product.

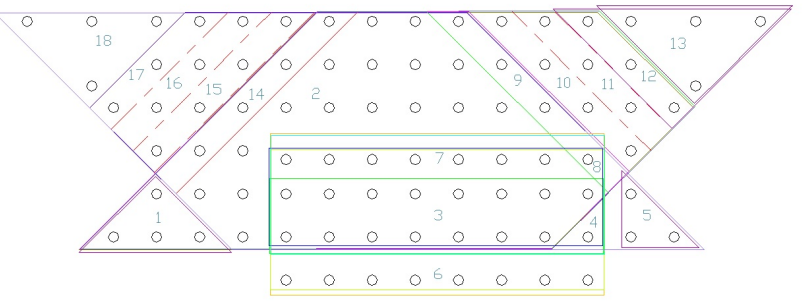

Figure 8: A gripper designed for handling all plies except the 0degree layers in the stack of figure 1 . Gripper consists of 18 zones that can be assembled to handle 22 ply geometries.

The resulting gripper after stagger reduction, shown in Figure 6, consisted of two sides, but the sectors where reduced from 18 to 6 (with +1 zone in an additional gripper for the 90 -degree layers). The UD prepreg material was only protected by backing paper on one side. Vacuum gripper technology was chosen, and the robot lifted the material on the paper side to avoid contamination of the material. The vacuum cups where $15 \mathrm{~mm}$ in diameter and evenly spaced to distribute the lifting force with focus on creating enough lift at the edges of the plies. The side in contact with the cutting table was bare, and one of the issues encountered was a problem with the tacky material sticking to the surface, making lifting difficult. One way to handle this problem was to slow down the lifting process, letting the robot lift the material in small incremental steps. As described above, the adhesion of the prepreg material is time dependent, thus making the removal of the material from a surface also time dependent. Also it was noted during the project that lifting plies straight up in the normal direction of the surface did not aid prepreg-to-surface separation. An alternative solution, to "peel" the prepreg of the cutting table lifting at an angle from the surface, was discussed. Although the zones aid in angled lifting the large gripper design limited the possibilities and this was not tested in the project. Also, a common way to handle unwanted sticking of prepreg to surfaces is to use protective plastics sheets. However, this introduces additional process step, namely the removal of the protective sheet before stacking and thus this solution was avoided.

After the end-effector picked a cut ply from the cutting surface the ply was moved to a device that created an initial separation between the prepreg and the backing paper. This separation is only created for part of the ply and needed for the automated removal of the backing paper. After the separation stage the ply was moved to the layup area where it was placed on the multistack in a direction directly nominal to the surface. This resulted in full ply-to-ply contact between placed and previous plies, risking air to be entrapped between the placed ply and the stack in the following steps. To ensure full adhesion before the backing paper was removed the robot performed a rolling operation using a roller assembled to the same end-effector that was used for the pick-and-place. The subsequent removal of the backing paper was done using the gripping device shown in Figure 9 mounted on the same end-effector as was used for the pick-and-place. To ensure that the backing paper was held firmly during the peeling a claw device was used in combination with a vacuum cup. After a certain number of layers had been fully assembled, the stack was covered with a rubber membrane and vacuum was drawn to remove entrapped air ensuring final product quality.
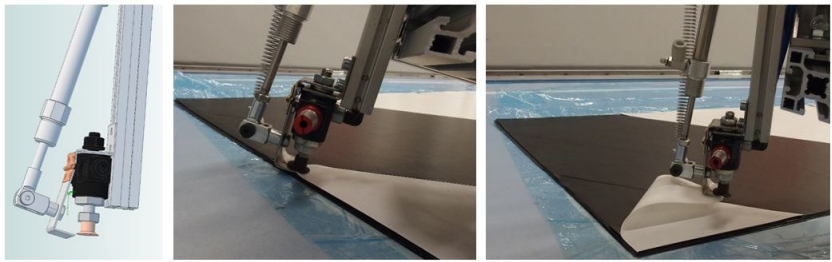

Figure 9: Left: The suction cup and claw for backing paper removal. Middle and right: The backing paper removal process.

\section{Dual Arm Demonstrator}

The dual arm demonstrator is the result from the first step of a research project carried out in collaboration between Linköping University and SAAB Aerostructures. The aim has been to develop a demonstrator for automated stacking prepreg plies to form a multistack using a pick-and-place approach. The demonstrated operations are highlighted with bold letters in the overall manufacturing process description in Figure 10. In later stages of the project a solution for forming, using the same dual arm robot will be developed and integrated to a fully automated manufacturing cell.

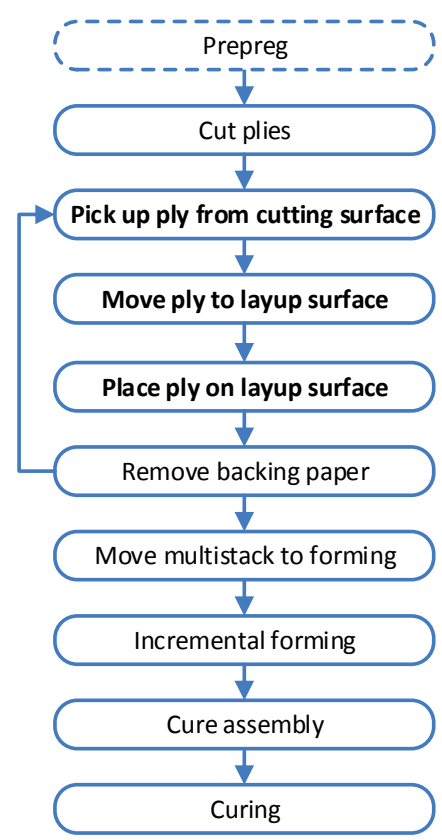

Figure 10: A concept for a fully automated manufacturing process. The operations marked with bold letters have been a part of the current demonstrator.

A Motoman SDA10 robot, shown in Figure 11, has been used for the demonstrator. The robot, with a handling capacity of $10 \mathrm{~kg}$ for each arm, picks up cut prepreg plies from a flat surface and stacks them in a multistack on another flat surface. The pickup step has been developed so that it is possible to pick one ply without affecting adjacent material, resembling the case when picking from a cutting machine surface. The prepreg material used is covered by backing paper on the side facing up from the pickup surface. The pick-andplace process has been developed so that it is possible to integrate the same type of automated removal of backing paper that is used in the single manipulator demonstrator above. However in the first stage of 
the project, presented here, the removal of prepreg backing paper was performed manually.

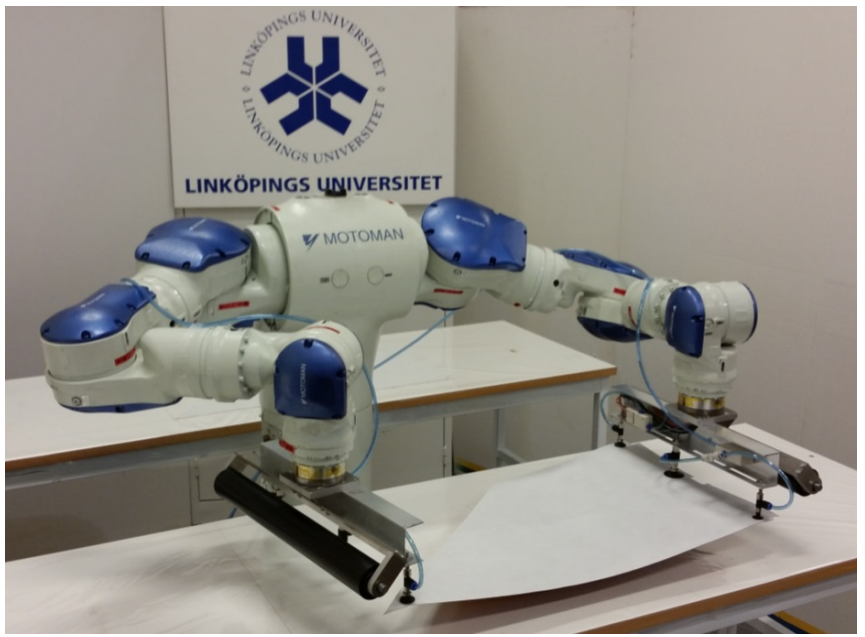

Figure 11: The dual arm robot equipped with the developed endeffectors holding a 45-degree prepreg ply with backing paper.

A primary focus for the project was to develop a solution that could handle a great variety of prepreg ply geometries, not only the ply geometries used for demonstrations. However, for demonstration purposes a demonstration product was selected. The demonstration product was a support structure for an aircraft component. The structure contains 12 plies of unidirectional carbon fiber prepreg. The plies covers the four major fiber orientations, $0^{\circ}, 45^{\circ},-45^{\circ}$ and $90^{\circ}$. The largest ply shape is approximately $400 \times 1100 \mathrm{~mm}$ and the plies are stacked in an arrangement that result in a 7 layer thick multistack.

The end-effectors, shown in Figure 12, include two separate functions, gripping and rolling. For the gripping function standard vacuum cups and vacuum ejectors are used. Since the grippers only are in direct contact with the prepreg backing paper the risks of contaminating the prepreg and resin buildup on the grippers are reduced. The backing paper has shown to help increase the rigidity of the plies. This aspect is particularly important in the dual arm solution where only a few interface points, in this case up to six vacuum cups, are used to lift the ply. The rolling function is used when a ply is placed on the multistack.
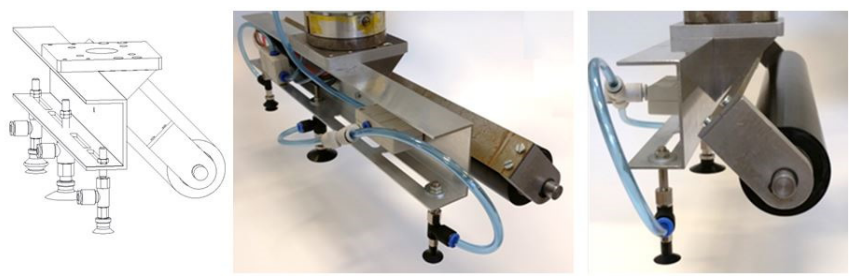

Figure 12: The end-effector design for the two end-effectors developed for the dual arm demonstrator. Left: CAD-drawing of an end-effector. Center and right: The realized end-effector.

The laydown sequence is illustrated in Figure 13. The prepreg ply, in this case a 45-degree ply, was placed in the correct position and one side of the ply was pressed in contact with the previous placed plies in the multistack by one of the end-effectors (A). This end-effector then changed from the gripping function to the rolling function (B).
In a coordinated movement between the two robotic arms the roller moved from the starting position to the other side of the ply at the same time as the other end-effector lowered the prepreg ply (C). With a short rolling distance left, the gripping end-effector released the prepreg ply (D \& E) so that the roller could access the last part of the ply. The rolling operation made sure that the prepreg plies adhered to the stack so that the backing paper could be removed without damaging the plies.
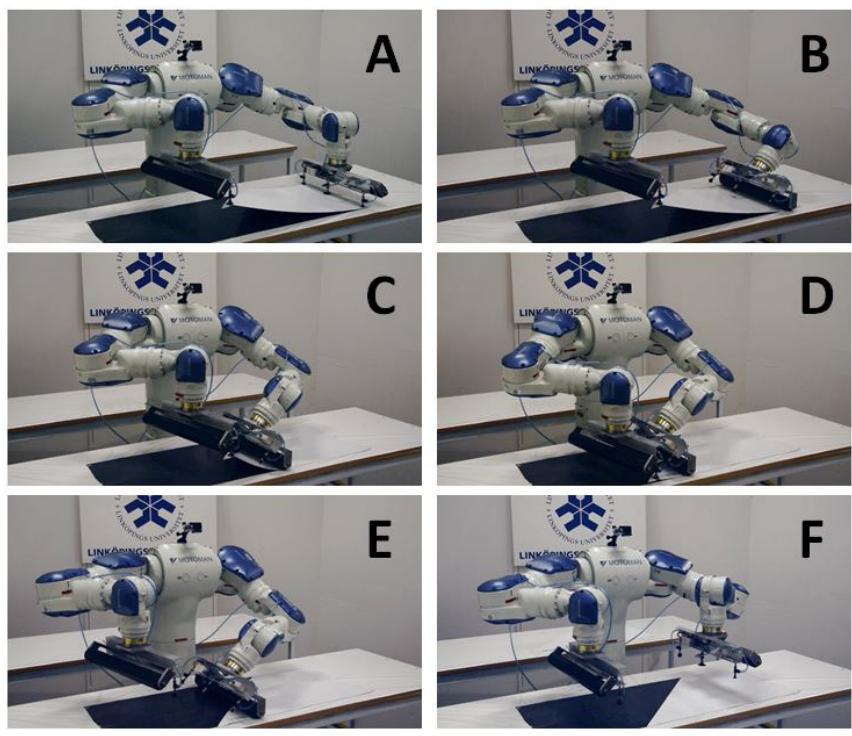

Figure 13: The laydown sequence of a 45-degree ply.

\section{Discussion}

For both concepts the positional accuracy of the placed plies proved sufficient to industry standards and both concepts were able to lay up multistacks with acceptable quality according to general industry criteria.

In the single manipulator case described above the end-effector became complex in order to provide flexibility i.e. to handle as many ply geometries as possible in the same gripper. The ability to handle many ply geometries therefore came at the cost of the end-effector being bulky, hampering maneuverability. The dual arm solution simplified end-effector design utilizing the ability to program the two arms relative positions as to handle variations in ply shapes. However the dual arm solution does not necessary have to be a dual arm robot as in the case above. The solution can just as well utilize two single manipulators, like the robot used in the single manipulator demonstrator, with synchronized movements. The use of two single manipulators can enlarge the working area, which in the case of the dual arm robot turned out to be rather limited. The seven axis configuration used in each arm of the dual arm robot might however give a possibility for more complex motions than the more common six axis configuration.

In the dual arm solution the material tend to hang down in between the two end-effectors. The hang down did not affect the layup of the plies used for the demonstration. However with increasing ply sizes this effect might have to be taken into account when designing the gripping function of the end-effectors.

Page 5 of 7 
Cutting the prepreg material with an unprotected surface facing down towards the cutting table generated strong adhesion between the prepreg and the cutting surface. The strong bond made it difficult to remove the plies after cutting. The possibility for synchronized relative movements between the end-effectors in the dual arm solutions could be used to make a peeling motion when removing the plies from the cutting table. Initial test have shown that a motion where one gripper starts to lift a ply at one outer edge and thereby peels the ply from the surface works much better than lifting the whole ply in directly nominal to the cutting surface.

This type of handling systems that have been demonstrated above can also be used for automated cutting and kitting of disposable materials that are used in prepreg manufacturing processes such as breathers, peel-plies and plastic films. The cutting and handling of disposable materials is today generally made manually and make up a considerable part of the total manufacturing time when manufacturing products based on prepreg materials.

\section{Conclusions}

Both demonstrators have successfully shown that it is possible to automatically stack plane laminates using a pick-and-place approach. Due to plies being assembled and due to ply stagger is it is necessary for automated pick-and-place concepts to handle a great variety of prepreg ply shapes. The two demonstrators have taken two separate approaches to solve this issue. The single manipulator demonstrator require a more complex end-effector design than the dual arm approach in order to handle several different ply shapes. The dual arm solution can employ simple end-effectors and the ability to reprogram their relative positions to handle geometrical variations. The dual arm approach also offers a possibility for peeling motions when picking up prepreg from a cutting surface. However, this can, to a limited extent, also be incorporated in a single end-effector solution. The peeling capabilities can also be used to create a successive laydown of the plies in combination with a rolling operation that make the plies adhere to the multistack and reduce the risk for entrapped air in-between the layers. For both demonstrators, keeping the backing paper has shown to be advantageous since it reduce the risk for contamination and provides a surface that is easy to grip using vacuum cups without resin buildups. The backing paper also provides extra rigidity to the prepreg plies which is good, especially in the dual arm case where a low number of interface points are used for gripping.

There is a need to automate handling of prepreg plies and stacking of plane laminates and the two demonstrators described in this paper have shown that it is possible to do that using simple, low-cost automation. Automating the processes of picking from a cutting table and stacking plane laminates can give a significant reduction in the needed manpower compared to today's manufacturing process.

\section{References}

1. Hale, J., "Boeing 787 from the Ground Up", Aero QTR_04.06, 2006.

2. Reinhart, G. and Straßer, G., "Flexible gripping technology for the automated handling of limp technical textiles in composites industry", Production Engineering 5(3): 301-306, 2011.

3. Löchte, C., Kunz, H., Schnurr, R., Dietrich, F., Raatz, A., Dilger, K. and Dröder, K., "Form-flexible Handling Technology for Automated Preforming", Presented at 19th intern. Conf. on Comp. Mat. (ICCM19), Canada, July 28 - August 2, 2013.
4. Ehinger, C. and Reinhart, G., "Robot-based automation system for the flexible preforming of single-layer cut-outs in composite industry", Production Engineering 8(5): 559-565, 2014.

5. Broetje Automation, "Composite handling system", http://www.broetje-automation.de/en/solutions-customerbenefit/equipment/composite-manufacturing/handling/, March 2015.

6. Ward, C., Bhatnagar, V., Potter, K., "Developing an Automated System for the Removal of Protective Films from Pre-Preg Material, to Remove a Manufacturing Bottleneck in Terms of Pick and Place Automation", Presented at SAMPE SETEC 13, Germany, September 11-12, 2013.

7. Advanced Composites Group Ltd, "ARMATURE Automation Project", Project presentation folder, 2011.

8. Black, S., "Automated cutting accelerates", http://www.compositesworld.com/articles/automated-cuttingaccelerates, Marc 2015.

9. Björnsson, A., "Enabling Automation of Composite Manufacturing through the Use of Off-The-Shelf Solutions", Licentiate thesis, Department of Manufacturing Engineering, Linköping University, Sweden 2014.

10. Campbell, F.C., "Manufacturing processes for advanced composites", (Oxford, Elsevier Advanced Technology, 2004), ISBN: 1856174158

11. Lukaszewicz, D.H.-.A., Ward, C., and Potter, K.D. , "The engineering aspects of automated prepreg layup: History, present and future", Composites Part B: Engineering, 43(3):9971009, 2012.

12. Buckingham, R. and Newell, G., "Automating the manufacture of composite broadgoods", Composites Part A: Applied Science and Manufacturing, 27(3):191-200, 1996.

13. Putnam, J., Seferis, J., Pelton, T., and Wilhelm, M., "Perceptions of prepreg tack for manufacturability in relation to experimental measures", Science and Engineering of Composite Materials, 4(3):143-154, 1995

14. Björnsson, A., Lindbäck, J. E., and Johansen, K., "Automated Removal of Prepreg Backing Paper - A Sticky Problem" SAE Technical paper 2013-01-2289, 2013, DOI: 10.4271/2013-012289

15. Dubois, O., Le Cam, J. and Béakou, A., "Experimental analysis of prepreg tack", Proceedings of the Society for Experimental Mechanics, Inc., 67:599-606, 2010.

16. Crossley, R., Schubel, P., and Warrior, N., "The experimental determination of prepreg tack and dynamic stiffness", Composites Part A: Applied Science and Manufacturing 43(3):423-434, 2012.

17. Stephan, J., and Seliger, G., "Handling with ice-the cryogripper, a new approach", Assembly Automation 19(4):332-337, 1999.

18. Kolluru, R., Valavanis, K.P., Steward, A., and Sonnier, M.J., "A flat surface robotic gripper for handling limp material", Robotics \& Automation Magazine 2(3):19-26, 1995.

19. Ozcelik, B., Erzincanli, F., and Findik, F., "Evaluation of handling results of various materials using a non-contact endeffector", Industrial Robot: An Int. J. 30(4):363-369, 2003.

20. Sjölander, J. and Åkermo, M.," Automated robot forming of stacked UD-prepreg - Experimental and numerical analysis“, Submitted to: Composites Part A, Submitted 2015.

Page 6 of 7 


\section{Contact Information}

The corresponding author of this article is:

Andreas Björnsson

Linköping University

andreas.bjornsson@liu.se

\section{Acknowledgments}

The research presented in the article is a part of the NFFP-program, funded by med Swedish Armed Forces, Swedish Defense Materiel Administration and Swedish Governmental Agency for Innovation Systems, and the Triple Use project funded by Swedish Governmental Agency for Innovation Systems as part of the

LIGHTer initiative. The work of master's students Filip Jöndell, Tina Nikko and Sofia Oskarsson is greatly acknowledged. 